\title{
An Enhanced Concept based Approach for user Centered Health Information Retrieval to Address Readability Issues
}

\author{
Ibrahim Umar Kontagora ${ }^{1}$, Isredza Rahmi A. Hamid ${ }^{2}$, Nurul Aswa Omar ${ }^{3}$ \\ Faculty of Computer Science and Information Technology, Universiti Tun Hussein Onn Malaysia (UTHM) ${ }^{1,2,3}$ \\ Batu Pahat, Johor Malaysia \\ Department of Computer Science, Niger State Polytechnic, Zungeru, Niger State, Nigeria ${ }^{1}$
}

\begin{abstract}
Searching for relevant medical guidance has turn out to be a general and notable task executed by internet users. This diversity of quantifiable information explorers indicates the enormous range of information needs and consequently, a key prerequisite for the development of clinical retrieval systems that would satisfy the clinical information desires of non-clinical professionals and their care givers. This study focused on designing an enhanced model for clinical consumers balanced based medical information retrievals and also proposed an improved system model that would provide simpler medical meanings for every clinical grammer(s) established on a clinical released documents and clinical search results online. We evaluated and compared the enhanced model with the current models in the clinical domain, namely, QLM ("Query Likelihood Model"), LSI ("Latent Semantic Indexing") and CBA ("Concept Based Approach") using MeSH, Metamap and UMLS databases. The outcomes gotten from the investigational study confirmed that, the Enhanced model (ECBA) managed to achieve 0.9145, 0.9170 and 0.9156 on MAP ("Mean Average Precision"), P@10 ("Precision@ @ 10") and NDCG@10 ("Normalized Discontinued Cumulative Gains @ 10") in that order. Hence, the superlative model to be deployed in addressing readability hitches is the Enhanced Concept Based method.
\end{abstract}

Keywords-Concept-based approach; medical discharge reports; clinical reports; query expansion; latent semantic indexing; query likelihood model

\section{INTRODUCTION}

Health information retrieval has become one of the most performed tasks on the internet today $[10,16]$. Additionally, it has become extremely difficult to cope with the speed of release of new researches in the biomedical domain [23]. Searching online channels for medical guidance has turn out to be a common performed task by individuals on the web. $[21,23]$. A fresh study piloted by United States shows that about 80 percent of online information seekers conduct internet search for their medical information $[10,30]$.

However, the incapability of the current approaches to address the diverse information desires for various classes of information seekers (clinical professional and nonprofessional), has made non-clinical professional and their care givers to further queries after reading through their displayed probe results online $[32,23]$. The previous researches targeted more on a specific group of information seekers with clinical acquaintance [26].

The Pursuit for medical guidance online has turn out to be a common and significant job executed by persons seeking for relevant health information on a specific disease $[10,23]$. The increasing number of online health information probe has driven several number of assessment crusades concentrating on clinical information retrievals [16]. Some of these assessments campaigns aimed at identifying and addressing the medical information needs of non-clinical professional and their assistants through their medical documents as they enlist for clinical attention [31].

Patient portal is defined as an efficient and effective means of providing patients with a 24 hours free access to personal health information from any destination online $[28,15]$. Different from the traditional personal medical records, patient portals are financed and taken care of by the portal administrators [18, 26, 29]. One of the major merits of patient portal is that, it allows patients to take control of the management of their health [32, 17]. However, patients' portals are predominantly available to outpatients [14]. Investigations have also revealed that, age is also one of the major contributory factor affecting the practice of patients portals, as there are more patronage on younger ages than the older ones [4, 5]. The work by [35] focused on evaluating the impacts and designing a patient portal for inpatient that would provide patients with a 24 hours free admittance to particular medical facts with internet connection from anywhere.

The work by [34] focused on the evaluation of user involvement as a key vital tool to the success of the design and implementation of a medical collaboration and communication platform aimed at improving medical care by medical team of clinicians. Even though medical information retrieval systems have the potential to enhance the safety and effectiveness of medical delivery, this potential is yet to be fully achieved [19, 31]. However, medical information retrieval systems require the full incorporation of friendly tools during their design that are context appropriate [7].

User centered design, user co-design and participatory design are the three main approaches adopted in involving users in user centered health information design [20, 24, 8]. Re-counted merits of users involvement in user centered 
health information systems designs include the design of enhanced information retrieval systems that would better address the apprehension problem faced by laymen patients and their care givers in discovering information mined from their displayed search results online [31, 8]. Despite these merits, user centered health information systems design remains challenging to attain, as a result of technical, communal, structural and cultural influences [24, 8].

Preceding studies on medical information retrieval discloses that patients are continually inquisitive of knowing what exactly is written on their medical discharge documents and clinical reports $[32,16]$. However, the unsatisfying concern is that, the medical tests are usually very proficient and tough to understand [20]. And for that, medical information retrieval becomes extremely admired as an efficient means of answering questions that might be asked by the patients [6]. Several assessment campaign communities have embarked on the conduction of domain-specific resources, organizing worldwide challenges / competitions / researches in the arena of biomedical information retrieval, in order to provide the needed information by medical experts and laymen patients $[25,31]$.

The core objective of this research study is to propose an improved system model and to design an enhanced approach using the concept based approach that would better address the apprehension problem faced by laymen patients and their care givers in discovering information mined from their displayed search results online. The proposed enhanced approach would provide simpler translations for all medical words found on the displayed search results online. The remaining sections of the paper are organized as follows. The reviewed related works is contained in Section 2, the proposed system model and improved approach are discussed in Section 3, the performance analysis is discussed in Section 4 and the conclusion of the work and recommendation for future work is contained in Section 5.

\section{RELATED WORK}

Previous related works have extracted and investigated data from the query logs of numerous commercial search engines [34]. However, the outcome of the investigation shows that, the concentration given to the creation of systems that meet up to the information desires of laymen patients is significantly low $[10,23]$. Additionally, this has significantly contributed to the high rise of apprehension issues faced by the non-clinical professionals while reading through their retrieved search results displayed online [21]. Work by [35] proposed that sufficient consideration should to be accorded to the information requests of various classes of information seekers.

The creation of consumer balanced information retrieval systems for laymen queries have proved difficult, as medical words apprehension still remained a major challenge from retrieved displayed search results by non-clinical professionals and their care givers $[25,16]$. Several clinical retrieval systems are unable to integrate program modules that would provide simpler words/ translation for all medical grammars found in clinical release documents and clinical search results online [21, 37].
The wide acceptance of user centered clinical systems was as a result of excellent and proficient medical care delivery and life touching excellent impact it renders to end-users [1, 2, 10]. In an attempt to address the apprehension problem faced by the non-clinical professional patients and their assistants or care givers, the development of enhanced algorithms that would determine the successes and failures of a consumer balanced clinical systems in respect to addressing readability problems were projected by [34, 25]. How information retrieval systems could improve health delivery and accessibility to quality well-being systems was the focus of the work by $[36,37]$.

Previous researches on medical document retrieval also reveals that the curiosity of patients having a clear picture of the content was as a result of the professional nature of how clinical release documents and clinical search results are written online. However, the medical texts are usually very professional and difficult to follow and as such, they still need to consult other medical experts for the meanings of the terms contained in the search results $[16,22,23]$. In an attempt to effectively answer these questions raised by patients, highly robust algorithms were proposed by the researchers [6]. In addition, previous studies have also revealed that, queries that do not reflect users' specific information needs failed to address readability issues [21].

The complete implementation of electronic health information systems in different context has attracted advanced researches in health information retrievals [1, 32, 33]. Earlier studies had shown that, the timely availability of readable and accurate Information had improved health delivery and clinical decisions [22, 27]. Additionally, the acceptance and full implementation of electronic health information system by clinicians, has also contributed to the entire successes recorded in the recent time in tackling apprehension problems faced by non-clinical professionals patients in mining information explored from their clinical release documents and clinical search results online $[10,11$, 36].

Despite the awareness of the existence of readability issues, previous researchers have continued to pay more attention in addressing medical practitioners' information needs $[33,20]$. There was limited attention to addressing the information needs of non-clinical professional patients and their assistants by providing simpler interpretations for all medical grammers found on clinical release documents and clinical search results online [12]. The primary aim of a consumer balanced retrieval system is to provide medical information seekers with a patient centered information [13, 3, 37].

\section{The PROPOSED ENHANCED CONCEPT BASED APPROACH}

The study proposed an enhanced method for consumer balanced health information retrieval systems that would address readability issues by providing simpler translations for all medical grammars seen on clinical release documents and clinical search results online. The enhanced method was improved by incorporating two special functions modules namely: the module for generating medical search queries in layman's forms and module for generating medical discharge 
documents in layman's forms. The proposed approach provides layman's translation for every medical term(s) found on a medical discharge document or medical search queries results online. More so, it also fully incorporate and implement two additional special controlled vocabularies modules namely: medical terms controlled module and vocabulary controlled module. They restricts the search terms in a launched search query to most specific terms (MST), in order to prevent vocabulary mismatched issues.

\section{A. A Model for the Proposed Enhanced Concept based Approach}

From the Model of the proposed enhanced method in Fig. 1, the system first of all prompts the user to input the information to be searched for. Upon inputting the requested search information, it does two things, firstly, is to launch a search query and secondly, is to send a notification message to the Medical Concept Free Module that a search query has been launched. Upon receiving such notification, it refers to the search query, search and extract all the most specific medical terms (MST) in the search query. The extracted most specific medical terms (MST) are then moved to the vocabulary controlled module where the synonyms of all the extracted most specific terms (MST) are searched for and extracted from the dictionary of the selected online dataset. At this point, both the extracted most specific terms (MST) from the launched search query and their extracted synonyms terms from the selected dataset are then expanded into the new search query. Finally, it displays the search results with their layman's translations to the end-users as shown in Fig. 1.

In addition, the proposed approach was enhanced to limit its search terms to only most specific medical terms in the launched search queries, as well as the synonyms of these terms extracted from the dictionary of the selected dataset, in order to prevent vocabulary mismatched issues. However, these was achieved by the implementation of two additional program modules namely the Medical Concept Free Module and the Vocabulary Controlled Modules. The former ensures that only most specific medical terms in a launched search query are extracted, while the later ensures that only the synonyms of the extracted most specific terms from the dictionary of the selected database are searched for, extracted and expanded into the new search query.

1) The significance of our proposed enhanced method is that, it provides simpler translations for all medical grammers/words found on a retrieved clinical release documents and clinical search results online. Also, for the specific purpose of ensuring that, the medical grammers/words enclosed in the two text fragments (input and output) are related, the medical terms controlled module, as well as the vocabulary controlled modules were also created and integrated into the proposed enhanced method. By so doing, the improved method better addressed the enquiries that non clinical professional patients and their assistants do seek after perusing through their clinical release documents and clinical search results online. More so, the performance of the our improved method with the current approaches were evaluated using data were extracted from the dictionary of Medical
Subject Heading, Metamap, Unified Medical Language System and Khresmoi project 6 datasets.

\section{B. The Enhanced Approach using Concept based Approach}

The study proposed an improved approach using the concept based approach that addresses readability issues by providing simpler interpretations for all clinical words/grammars seen on patients' clinical release documents and clinical probe results online. The improved approach using the concept based approach was enhanced by incorporating two special functions modules namely: the module for generating clinical probe results in simpler forms and module for generating clinical released documents in simpler forms. The improved method which comprises of lines numbers $1-16$ will also fully implement the two additional incorporated controlled vocabularies in order to avoid vocabulary mismatched issues.

The integrating strategy for the proposed approach is as shown in Fig. 2, where input $N$ is the counts of words generated from the core search, $\mathrm{C}$ is concept terms and $\mathrm{K}$ is expansion terms. $S Q$ represents search query and $C C_{n}$ represents the $n t h$ concept, $K$ signifies the counts of extension words, and $E T_{k}$ symbolizes the kth extensions words. The kth is the tailed extensions words in an expansion query and the sign \# signifies vacuum space. The dual quote symbols signify that the word contained will surface successively. The Medical Concept Free Module ensures that only most specific medical terms in a search query are searched for and extracted. Finally, inputted search information is end-users information request entered into the search query as shown in Fig. 2.

For the specific purpose of providing simpler translations for all the clinical grammars/words established on retrieved clinical released documents and clinical probe results online, the module for generating clinical probe results in simpler form provides the simpler interpretations for all the clinical grammars/words seen on the displayed clinical probe results online. While, the module for generating clinical released documents in simpler form provides the simpler interpretations for all the clinical grammars/words found on the displayed clinical released documents as shown in Fig. 3 and 5. The improved method was implemented in two levels for the purpose of medical document retrievals. (i) By searching and extracting only the labeled most specific concepts terms in a search query and their synonyms from the dictionary of the selected dataset and (ii) by providing layman's translations for all the medical grammars/words found on the retrieved clinical released documents and clinical search results online. Hence, it better address the information desires of non-clinical patients and their assistants.

\section{Module for Generating Clinical Probe Results in Simpler form}

Specifically, the creation and generation of clinical released documents and clinical search results in simpler form were achieved using Fig. 3 and 5 respectively. Fig. 3 is the module for generating Clinical Probe Results in Simpler form. It consists of lines numbers 17 to 29 . It is designed in such a way that once a clinical probe query result is displayed, it 
searches and provides the simpler interpretations for all the clinical grammars seen on the displayed clinical probe results online as contained in Fig. 4.

In Fig. 3, replace grammar refers to those found medical grammars in the clinical probe results whose simpler concepts are to be provided, @grammar refers to stored patients layman's terms in the dictionary of the selected dataset. Status gives the position of the search and excode tells the number of records found. However, this function provides the simpler interpretations for the entire clinical grammars/words seen on the clinical probe results.
Fig. 4 is the pictorial view of a generated output of a clinical query search result in Layman's/simpler Form generated by Fig. 3. The simpler grammar for such medical grammars can be gotten by clicking on the command button for layman's concept on the application web page for the proposed enhanced approach. Once a clinical probe result is displayed online, Fig. 3 searches and provides the simpler interpretations for the entire clinical grammars found on the displayed clinical probe result as could be seen in Fig. 4.

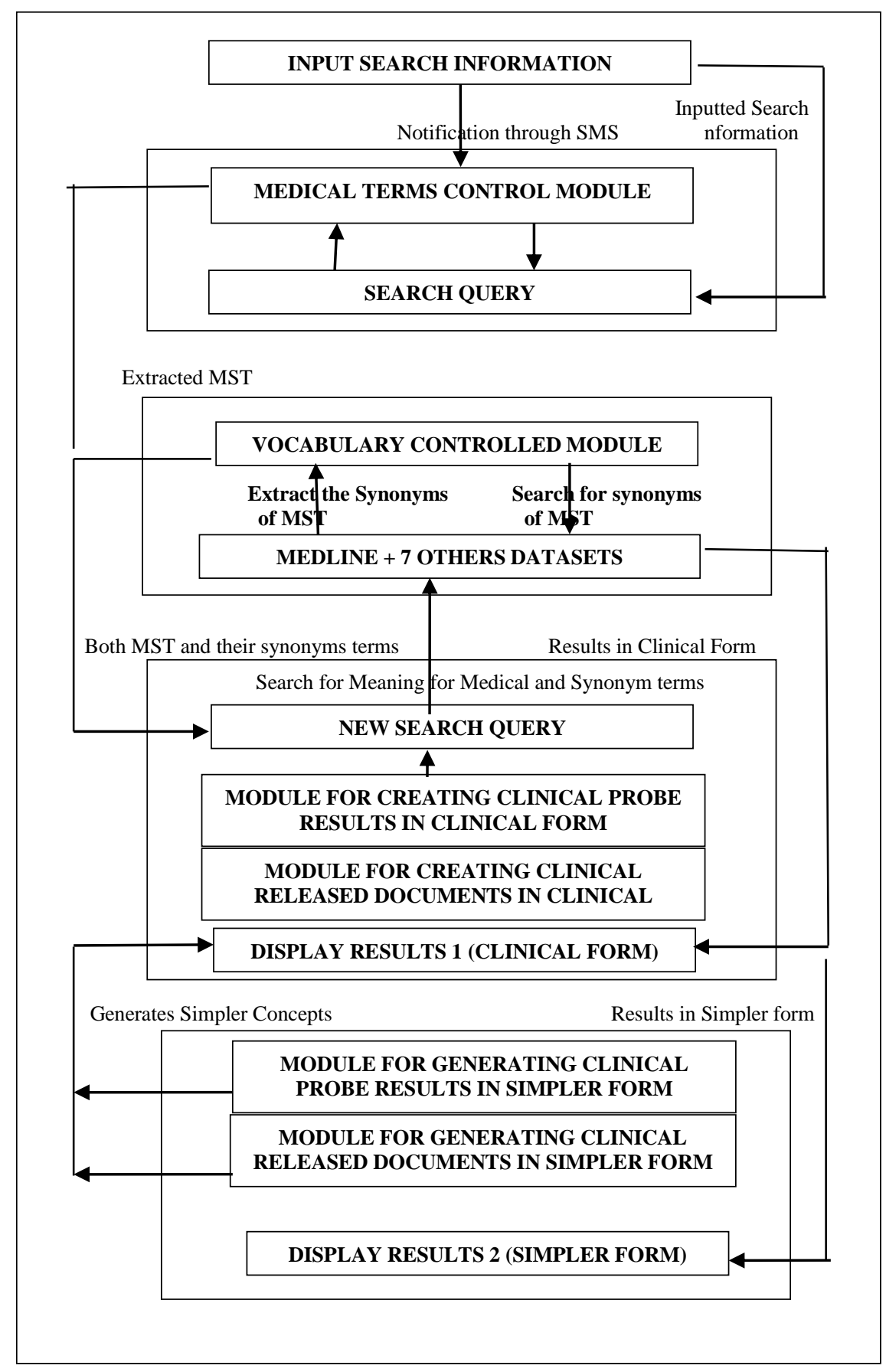

Fig. 1. A Model for the Proposed Enhanced Concept based Approach. 


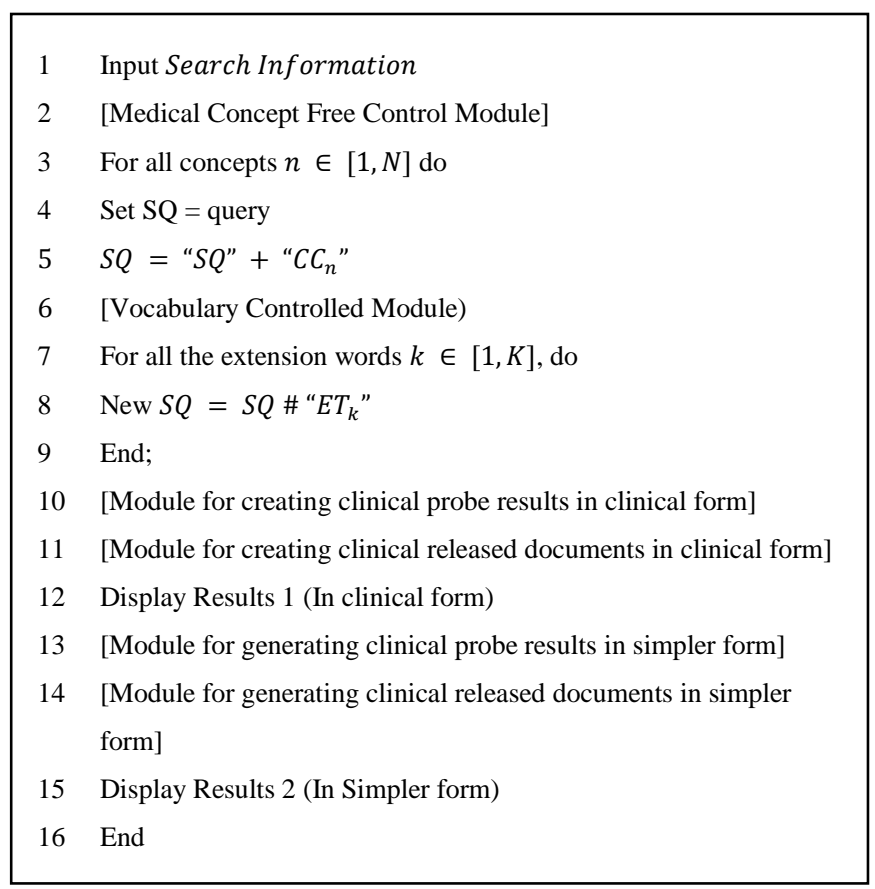

Fig. 2. The Enhanced Concept based Information Retrieval Approach.

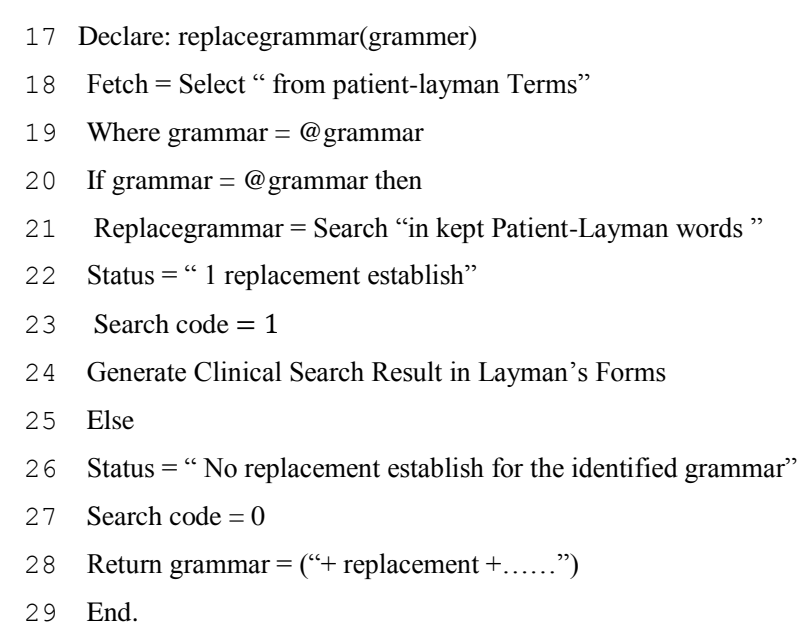

Fig. 3. The Module for Generating Clinical Probe Results in Simpler Form.

\section{Module for Generating Clinical Released Documents in Simpler Form}

In order to generate the simpler interpretations for every medicinal terms seen on a retrieved clinical released documents online, Fig. 5 was created. Fig. 5 is the module for generating clinical released documents in simpler forms. It comprised of line numbers. 30 - 42. It is designed in such a way that, once a clinical released document is displayed online, it searches, locate and provide the simpler interpretations for the entire medical grammars/words found on the displayed clinical released document online. By so doing, it better address the information desires of non-clinical professionals and their assistants as they explore information contained in their clinical released documents online.

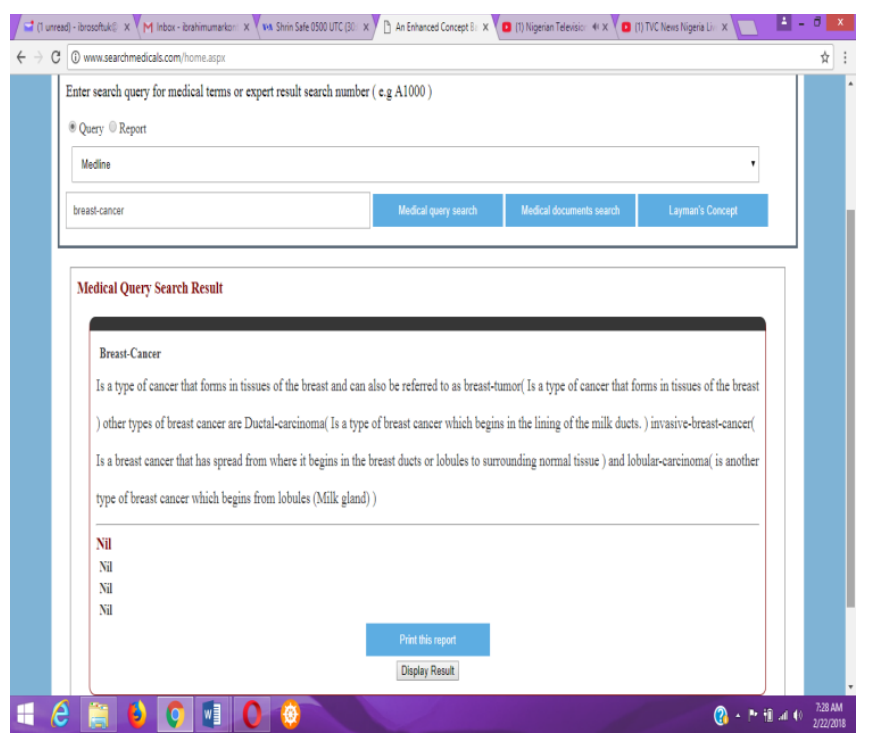

Fig. 4. The Pictorial View of Clinical Probe Result in Simpler Form.

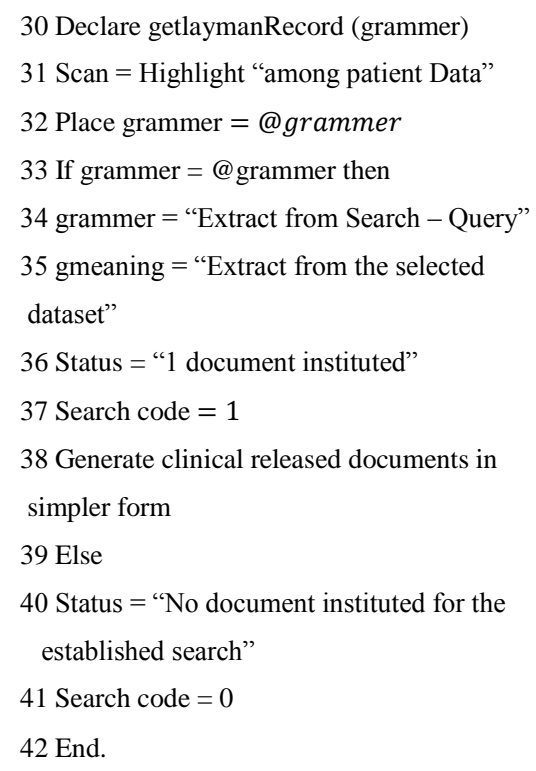

Fig. 5. The Module for Generating Clinical Released Documents in Simpler Form.

In Fig. 5, getlaymanRecord refers to search for layman grammar, grammar means the medical terms found in a medical discharge document, @grammar refers to stored grammars in the selected dataset, while gmeaning stands for the meaning of grammars found in a search query. Status gives the position of the search and excode tells the number of records found.

Fig. 6 is the Pictorial View of a generated output of a clinical released document in simpler form generated by Fig. 5. Once a clinical released document is being displayed online, it searches and provides the simpler interpretations for all the clinical grammars found on the displayed clinical released document as displayed in Fig. 6: 


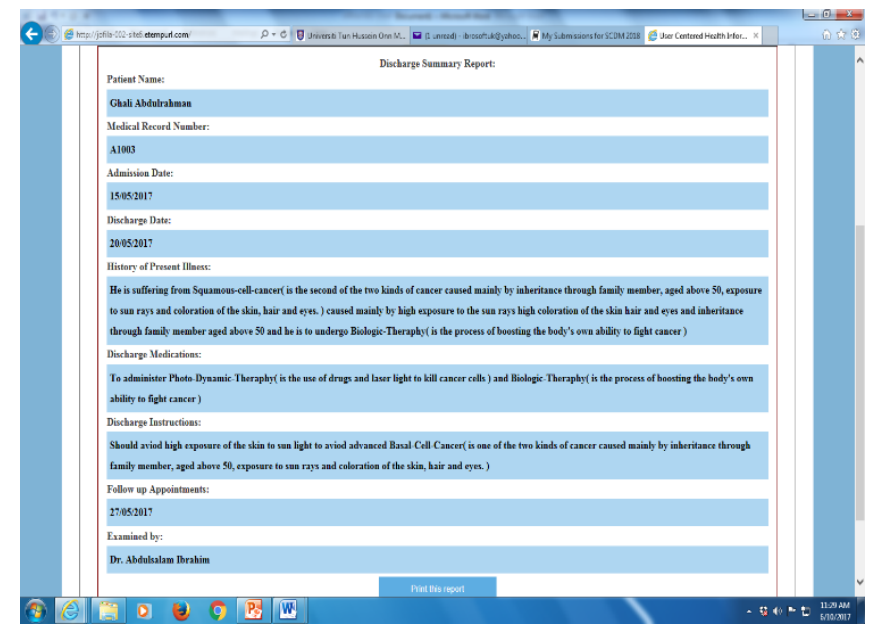

Fig. 6. The Pictorial View of Clinical Released Document in Simpler Form.

\section{Performance Analysis}

This section presents the experimental setup, dataset and performance parameters used in evaluating the enhanced method using the concept based approach (ECBA) and the current methods namely the latent semantic indexing (LSI), the concept based approach (CBA) and the query likelihood model (QLM) and.

\section{A. Experimental Setup}

The experimental setup was carried out using HTMLHyper-Text Mark-up Language, C\#.net, JavaScript and CSSnc -Cascading Style Sheet using Windows 7 operating system with Intel (R) Core i7 processor, 3.40GHZ and 4Gigabytes Random Access Memory (RAM). HTML was used for designing the web application structure and C\#.Net was used for creating and activating the application structure functionalities. JavaScript was used for making the web application dynamic and interactive and CSSnc was used for the beautification of the application looks.

\section{B. Dataset}

The study used Khresmoi Project 6, Metamap, MeSH and UMLS datasets in evaluating the systems performance. The datasets coverage is wide and comprehensive in the biomedical and health domain. All information in the pool were gotten from several online and free web databases among which are Diagnosia7 and Clinical.gov [23].

\section{Performance Metrics}

The performance of the current methods and the Enhanced Concept Based Approach were evaluated using 3 performance metrics namely:

a) $\mathrm{P} @ 10$ (Precision at 10 documents)

P@10 computes the fraction of applicable documents at each ten (10) recalls. It can be computed as $P @ 10=$ $\frac{(A) 10}{(A+B) 10}$ with $P$ being the Fraction of Applicable Documents Recovered at every ten (10) recalls, $A$ is Recovered Applicable Documents and $B$ is Recovered Non applicable Documents [9]. b) NDCG@10 (Normalized Discounted Cumulative Gain at 10 documents)

NDCG@10 calculates the collective achievement at separate point for a selected figure of $p$ for all the significant document in the probe [9]. NDCG@10 is computed as $N C D G_{p}=\frac{D C G_{p}}{I D C G_{p}}$ where $I D C G_{p}=$ $\left(I D C G_{p}\right)=\sum_{i=1}^{R E L} \cdot \frac{2^{\text {reli }}-1}{I D C G p} \cdot R E L$ signify the number of significant documents, $D C G_{p}$ is used to highlight extremely significant documents seeming promptly in the outcome list [9]

c) MAP (Mean Average Precision)

MAP calculates the Mean Average Precision of applicable documents recovered from a probe. MAP is computed as $M A P=\frac{1}{N} \sum_{J=1}^{N} \cdot \frac{1}{Q j} \sum_{i=1}^{Q j} \cdot P\left(d o c_{i}\right)$ where, $Q_{j}$ is amount of applicable documents for probej, $N$ is amount of probes and $P\left(d o c_{i}\right)$ is accuracy value at $i$ th applicable document [9].

\section{RESUlt AND DisCUSSIONS}

The performance of our enhanced method was evaluated using the performance parameters namely: MAP ("Mean Average Precision"), NDCG@10 ("Normalized Discounted Cumulative Gain at 10 documents") and P@10 ("Precision at 10 documents"). We compared the enhanced method with the current methods namely: LSI ("Latent Semantic Indexing"), CBA ("Concept Based Approach") and QLM ("Query likelihood Model"). The novelty of the enhanced method is that, it concentrates more in solving grammar apprehension issues faced by non-clinical patients and their assistants by providing simpler terms for every clinical grammars found on the displayed clinical released documents and clinical probe results online.

The results outcome contained in Table I and Fig. 7 revealed that, the enhanced method managed to achieve $91 \%$ accuracy in all the metrics, as compared to existing Concept Based Approached (CBA) which scored 80\%, Query Likelihood Model (QLM) scored 77\% and the Latent Semantic Indexing (LSI) scored $73 \%$ in all the four datasets. This clearly indicates that, the enhanced method, better addressed the clinical information desires of non-clinical professionals and their assistants as it provides simpler translations for the entire clinical grammars found on clinical released documents and clinical probe results online.

Sample data were extracted from the four datasets used namely: Medical Subject Heading (MeSH), Metamap, Khresmoi project6 and Unified Medical Language System (UMLS) for the precise task of evaluating the performance of the current methods and the enhanced method. The enhanced method was developed in a manner that, it provides simpler translations for the entire clinical grammars seen on displayed clinical released documents and clinical probe results online. The outcome of the simulation results obtained using the sample data extracted randomly from the four datasets used in the experiment is as shown in Fig. 7 and Table I: 


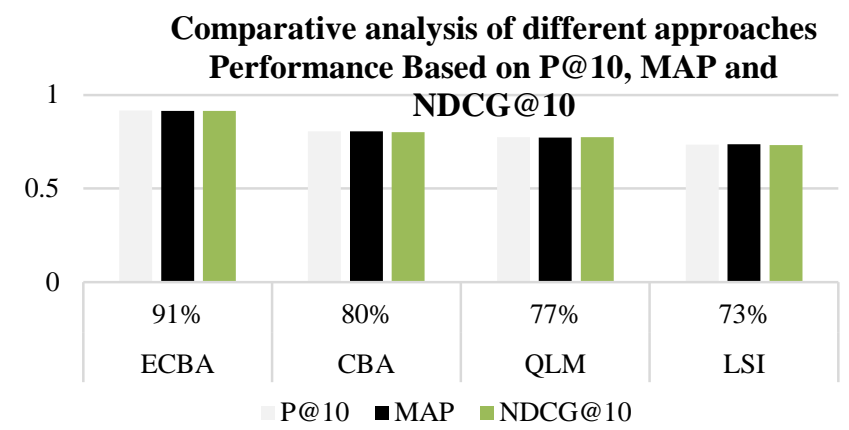

Fig. 7. Comparative Analysis of different Approaches Centered on MAP, NDCG@10 and P@10 Values.

TABLE I. A AMPLE SimUlATION RESUlts FOR All THE APPROACHES SHOWING THEIR ACCURACY PERCENTAGES USING THE MeSh DATASET

\begin{tabular}{|l|l|l|l|l|}
\hline \multirow{2}{*}{ APPROACH } & \multirow{2}{*}{$\begin{array}{l}\text { ACCURACY } \\
\text { (All Metrics) } \%\end{array}$} & \multicolumn{3}{|l|}{ PERFORMANCE METRICS } \\
\cline { 3 - 5 } & & P@ 10 & MAP & NDCG@ 10 \\
\hline ECBA & $91 \%$ & 0.9170 & 0.9145 & 0.9156 \\
\hline CBA & $80 \%$ & 0.8058 & 0.8048 & 0.8020 \\
\hline QLM & $77 \%$ & 0.7746 & 0.7729 & 0.7743 \\
\hline LSI & $73 \%$ & 0.7336 & 0.7366 & 0.7313 \\
\hline
\end{tabular}

Fig. 7 is the pictorial representation of the simulation results in Table I showing the performance of the current methods (QLM, CBA and LSI) and the enhanced method.

The experimental outcomes contained in Table I and Fig. 7 indicates that the enhanced method obtained a Precision@10 score of 0.9170, MAP score of 0.9145 and NDCG@10 score of 0.9156 . Whereas, the three current approaches namely: Query Likelihood Model (QLM) obtained a Precision@10 score of 0.7746 , MAP value of 0.7729 and NDCG@10 score of 0.7743 , Latent Semantic Indexing (LSI) scored P@10 value of 0.7336 , MAP score of 0.7366 and NDCG@10 value of 0.7313. And finally, the existing Concept Based Approach (CBA) scored a Precision@10 score of 0.8058, MAP value of 0.8048 and NCDG@10 value of 0.8020 . The results indicates that the enhanced method better addressed the grammar apprehension problem faced by non-clinical professionals and their assistants while perusing through their clinical released documents and clinical search results online.

The enhanced method works statistically based on the integrating strategy: For all concepts $n \in[1, N] \operatorname{do}$, Set $\mathrm{SQ}=$ query, that is, the entire medical grammars/words found in the search query would be searched for and extracted. $S Q=$ "SQ" + "CC ${ }_{n}$ ", For the entire extension grammarsk $\in[1, \mathrm{~K}]$, select all most specific concept terms. New $S Q=$ $\mathrm{SQ} \#$ " $\mathrm{ET}_{\mathrm{k}}$ ". $N$ is the amount of grammars gotten from the core query, $S Q$ signifies search query and $C C_{n}$ represents the $n$th concept. $K$ Signifies the amount of growth words while $E T_{k}$ signifies the $k$ th extension terms. The kth is the last extension concept in an extended query and the sign \# signifies universal words. And the duo quote marks specifies that the thread contained must appear successively. The statistical analysis also revealed that, in every 10 retrieved medical documents, the Enhanced Concept Based Approach (ECBA) precisely scored $0.9170 \quad(91 \%)$ as retrieved appropriate and comprehensible documents in relation to P@10, as against the existing Concept Based Approach (CBA) which scored 0.8058 (80\%), Query Likelihood Model (QLM) scored 0.7746 (77\%) and finally the Latent Semantic Indexing (LSI) which scored $0.7336(73 \%)$. In relation to NDCG@10, ECBA attained 0.9156 (91\%), QLM scored $0.7743(77 \%)$, LSI $0.7313(73 \%)$ and CBA $0.8020(80 \%)$ as appropriate and understandable retrieved documents and lastly in reference to MAP, ECBA Scored 0.9145 (91\%), QLM scored $0.7729(77 \%)$, LSI scored $0.7366(73 \%)$ and CBA scored $0.8048(80 \%)$.

\section{CONCLUSION}

The investigational results attained in Fig. 7 and Table I obviously revealed that, in every $10(100 \%)$ retrieved documents from a search query, the Enhanced Concept Based approach (ECBA) presents $91 \%$ as relevant and readable documents. While Latent Semantic Indexing (LSI), Query Likelihood Model (QLM) and the Concept Based Approach presents $73 \%, 77 \%$ and $80 \%$ as relevant and readable documents respectively. The logical reasons behind these outcomes gotten by ECBA, CBA, QLM and LSI can be elucidated by the results in Fig. 7 and Table I, where the proposed enhanced approach incorporated program modules that provides simpler interpretation for every clinical grammar found on the displayed clinical released documents and clinical probe results online.

The Enhanced method proved to better address the clinical grammar apprehension issues stumble upon by non-clinical professional patients and their assistants while reading through their clinical released documents and clinical search results online, as $91 \%$ of every retrieved information by the Enhanced method are relevant and readable, as against the Latent Semantic Indexing (LSI) 73\%, Query Likelihood Model (QLM) 77\% and Concept Based Approach (CBA) 80\%. The Enhanced method outweighs the Latent Semantic Indexing, the Query Likelihood Model and the Concept Based Approach by $11 \%$ to $18 \%$ in respect to Precision@10 NDCG@10 and MAP values. These improved outcomes was realized due to the ability of the Enhanced method to provide simpler interpretation for every clinical grammar found on the clinical released documents and clinical search results online. The study recommends that advance work on this research work should comprise of designing models and algorithms that would address clinical grammar apprehension issues faced by non-clinical professionals and their assistants on retrieved videos, images and audios.

\section{ACKNOWLEDGMENT}

The authors wish to express their profound appreciation and gratitude to the management of Universiti Tun Hussein Onn Malaysia (UTHM) for funding the research. The research was funded under UTHM TIER-1 Grant with vot number H107 and Ministry of Education Malaysia under FRGS Grant with vot number K047. 


\section{REFERENCES}

[1] H. Ayatollahi et al., "Factors influencing the use of IT in the emergency department". A qualitative study, Health Inf. J. 16 (3) (2010) 189-200.

[2] I. R. Bardhan and M. F. Thouin, "Health information technology and its impact on the quality and cost of healthcare delivery, Decis". Support Syst. 55 (2) (2013) 438-449.

[3] J. Bassi and F. Lau, "Measuring value for money". A scoping review on economic evaluation of health information systems, J. Am. Med. Inform. Assoc. 20 (4) (2013) 792-801.

[4] J. Barron et al., "Exploring three perspectives on feasibility of a patient portal for older adults, Stud”. Health Technol. Inf. 202 (2014) 181-184.

[5] S. Brahmandam et al., "Willingness and ability of older adults in the emergency department to provide clinical information using a tablet computer, J". Am. Geriatr. Soc. 64 (11) (2016) 2362-2367.

[6] C. Bellegarda et al., "Concept-based medical document retrieval: THCIB at CLEF eHealth lab 2013 task 3". In: Proceedings of the ShARe/CLEF eHealth Evaluation Lab. (2013).

[7] M. C. Beuscart-Zéphir et al., "The human factors engineering approach to biomedical informatics projects". State of the art, results, benefits and challenges, Yearb. Med. Inf. 10 (2007) 9-27.

[8] K. Cresswell et al., "Anything but engaged". User involvement in the context of a national electronic health record implementation, Inform. Prim. Care 19 (4) (2011) 191-206.

[9] L. Dybkjaer et al., "An Overview of evaluation methods". Evaluation of Text and Speech Systems in TREC Ad-hoc Information Retrieval and TREC Question Answering. 2007, Springer, Dordrecht, the Nethe

[10] Kontagora, I.U., Hamid, I.R.A. and Omar, N.A., "An Enhanced Concept based Approach for User Centered Health Information Retrieval to Address Presentation Issues". In International Journal of Advanced Computer Science and Applications (IJACSA), 10(2), pp 232 - 242, 2019. http://dx.doi.org/10.14569/IJACSA.2019.0100131.

[11] E. W. Ford et al., "Resistance is futile: but it is slowing the pace of EHR adoption nonetheless, J. Am". Med. Inform. Assoc. 16 (3) (2009) 274281.

[12] D. T. Ford, "Are electronic health records the future of dental practice?". J. Calif. Dent. Assoc. 43 (5) (2015) 238.

[13] A. Fuad and C. Y. Hsu, "High rate EHR adoption in Korea and health IT rise in Asia, Int”. J. Med. Inf. 81 (9) (2012) 649-650.

[14] S. R. Greysen et al., "Tablet computers for hospitalized patients". A pilot study to improve inpatient engagement, J. Hosp. Med. 9 (6) (2014) 396-399.

[15] T. Irizarry et al., "Curran, Patient portals and patient engagement". A state of the science review, J. Med. Internet Res. 17 (6) (2015) e148.

[16] N. Ksentini et al., "Miracl at CLEF 2014: eHealth information retrieval task". In: Proceedings of the ShARe/CLEF eHealth Evaluation Lab.

[17] C. S. Kruse et al., "The effect of patient portals on quality outcomes and its implications to meaningful use: asystematic review, J.Med". Internet Res. 17 (2) (2015) e44.

[18] C. S. Kruse et al., "Patient and provider attitudes toward the use of patient portals for the management of chronic disease: a systematic review, J. Med". Internet Res. 17 (2) (2015) e40.

[19] A. L. Kellermann and S. S. Jones, "What it will take to achieve the asyet-unfulfilled promises of health information technology, Health Aff". (Millwood) 32 (1) (2013) 63-68.

[20] A. Kushniruk and C. Nøhr, "Participatory design, user involvement and health IT evaluation, Stud". Health Technol. Inform. 222 (2016) 139151 .
[21] K. Dramé et al., "Query Expansion using External Resources for Improving Information Retrieval in the Biomedical Domain”. In CLEF (Working Notes), 2014, (pp. 189-194).

[22] J. King et al., "Clinical benefits of electronic health record use". National findings, HealthServ.Res.49 (1pt.2), (2014) 392-404.

[23] I. U. Kontagora and I. R. A. Hamid, "Comparative Studies of Information Retrieval Approaches in User-Centered Health Information System”. In: Ghazali R., Deris M., Nawi N., Abawajy J. (eds) Recent Advances on Soft Computing and Data Mining. SCDM 2018. Advances in Intelligent Systems and Computing, vol 700. Pp $171-180$. Springer, Cham.

[24] A. Lee et al., "Root cause analysis of serious adverse events among older patients in the Veterans Health Administration, Joint Comm". Res. 40 (6) (2014) 253-262.

[25] L. Goeuriot1 et al., "ShARe/CLEF eHealth Evaluation Lab 2015, Task 3". Information Retrieval to Address Patients' Questions when Reading Clinical Reports.

[26] M. Maher et al., "A novel health information technology communication system to increase caregiver activation in the context of hospital-based pediatric hematopoietic cell transplantation". A pilot study, JMIR Res. Protoc. 4 (4) (2015) e119.

[27] L. Nguyen et al., "Electronic health records implementation". An evaluation of information system impact and contingency factors, Int. J. Med. Inf. 83 (11) (2014) 779-796.

[28] Office of the National Coordinator, What is a Patient Portal?, "Available from: professionals/faqs/what-patient-portal"

[29] F. Pinciroli and C. Pagliari, "Understanding the evolving role of the Personal Health Record, Compute". Biol. Med. 59 (2015) 160-163.

[30] E. Papoutsi et al., "Patient and public views about the security and privacy of electronic health records (EHRs) in the UK". Results from a mixed methods study, BMC Med. Inform. Decis. Mak. 15 (1) (2015) 1.

[31] A. H. Pollack et al., "Leveraging physicians and participatory design to develop novel clinical information tools". AMIA Annual Symposium Proceedings/AMIA Symposium AMIA Symposium. 2016 (2016) 10301039.

[32] M. Rigby et al., "Patient portals as a means of information and communication technology support to patient-centric care coordinationthe missing evidence and the challenges of evaluation". A joint contribution of IMIA WG EVAL and EFMI WG EVAL, Yearb. Med Inf. 10 (1) (2015) 148-159.

[33] W. J. Rudman et al., "Integrating medical and dental records". A new frontier in health information management, J. AHIMA 81 (10) (2010) 36-39.

[34] J. J. Rodrigues et al., "Analysis of the security and privacy requirements of cloud-based electronic health records systems, J. Med". Internet Res. 15 (8) (2013) e186.

[35] H. Suominen et al., "The Proceedings of the CLEFeHealth2012 - the CLEF 2012 Workshop on Cross-Language Evaluation of Methods, Applications, and Resources for eHealth Document Analysis". (2015), NICTA.

[36] T. Schleyer et al., "Advancing oral medicine through informatics and information technology". A proposed framework and strategy, Oral Dis. 17 (s1) (2011) 85-94.

[37] W. Shen et al., "An investigation of the effectiveness of concept-based approach in medical information retrieval GRIUM @ CLEF2014eHealthTask 3". In Proceedings of the ShARe/CLEF eHealth Evaluation Lab, (2014). 